\title{
Optimal Vertical Jump of a Human
}

\author{
Mahdokht Ezati, Borna Ghannadi, Naser Mehrabi, John McPhee \\ Systems Design Engineering, University of Waterloo \\ ON N2L 3G1, Waterloo, Canada \\ mezati@uwaterloo.ca; bghannad@uwaterloo.ca; nmehrabi@uwaterloo.ca; mcphee@uwaterloo.ca
}

\section{Extended Abstract}

Recent research studies in biomechanics have demonstrated that the human central nervous system (CNS) coordinates body motions by minimizing some physiological cost functions [1]. The main goal of this research is to simulate an optimal human vertical jump by exploiting this principle. To this end, first, a simplified multibody model of a human has been developed and its dynamics equations have been determined. Then, to point up the key role of the toe joint in human jump, a toeless model has also been developed. The objective is to compare the toeless model with the main model, in terms of the jump height, jump style and joint torques. The joint torques of both models have been optimized to achieve an optimal vertical jump, employing an optimal control method.

The considered models are planar in this study. The main model is composed of five bodies including the toe, forefoot, shank, thigh and HAT (head-arms-trunk) while the toeless model does not have the toe and consequently includes only four bodies. To model the contact during the pre-flight phase, it is assumed that the toe body is fixed to the ground in the main model while the forefoot is fixed to the ground in the toeless model. In both models, the vertical jump is assumed to be divided into two phases, i.e. pre-flight and flight.

In the main model, during the pre-flight phase, the leg moves from the stance state to the heel-off motion and during the flight phase, the leg moves from the toe-off motion towards the take-off motion. However, in the toeless model, during the pre-flight phase, the foot remains fixed to the ground and during the flight phase, the foot leaves the ground and the model moves towards the take-off motion. In both models, flight phase will begin when the ground reaction forces and moment become zero and during the flight phase, the HAT has 3 degrees of freedom (DOF) with respect to the ground and thereby freely moving in the sagittal plane.

To determine analytical dynamics of the phases, the multibody models have been simulated in a physical modelling and simulation software, MapleSim. Then, the dynamics equations have been exported to MATLAB software with the aim of optimizing joint torques that yield the highest vertical jump. To solve the optimization problem, the essential constraints have been taken into account based on the human joint limitations and the cost functions have been defined with the aim of maximizing the vertical velocity of the models at the end of pre-flight phase, and maximizing the height of the models at the end of flight phase. Finally, the optimized simulation results of the models have been compared. To solve the optimal control problem, the GPOPS-II toolbox in MATLAB is used. GPOPS-II carries out optimization based on the orthogonal collocation method, which is a computationally efficient direct optimization method in optimal control theory [2]. The main reason behind choosing GPOPS-II is its capability to employ orthogonal collocation method along with nonlinear optimization on multi-phase optimal control problems, and this is considerably practical in biomechanics simulations [3].

\section{References}

[1] N. Mehrabi, R. S. Razavian, and J. McPhee, "A Physics-Based Musculoskeletal Driver Model to Study Steering Tasks," J. Comput. Nonlinear Dyn., vol. 10, no. 2, pp. 1-8, 2015.

[2] A. V. Rao, "A survey of numerical methods for optimal control," Adv. Astronaut. Sci., vol. 135, no. 1, pp. 497-528, 2009.

[3] N. Mehrabi, B. Ghannadi, R. Sharif Razavian, and J. McPhee, "Predictive Simulation of Reaching Moving Targets using Nonlinear Model Predictive Control," Front. Comput. Neurosci., vol. 10, pp. 1-12, 2017. 